\title{
Optimum Power Loss Analysis of Radial Magnetic Bearing using Multi-Objective Genetic Algorithm
}

\author{
Santosh shelke \\ $\mathrm{PhD}$ scholar \\ National Institute of technology, Warangal. \\ Andhra Pradesh (India).
}

\author{
R.V.Chalam \\ Professor \\ National institute of technology, Warangal \\ Andhra Pradesh (India)
}

\begin{abstract}
In this paper, weight optimization of radial magnetic bearing (RMB) for varying poles has been carried out using multiobjective genetic algorithms (MOGAs). The total weight of $\mathrm{RMB}$ and copper loss has been selected as the minimization type objective function. The maximum space available, saturation flux density, the maximum current densities that can be supplied in the coil and the load to be lifted have been chosen as constraints. The coil space radius, pole tip radius, radial length of coil and number of poles has been proposed as design variables. Apart from the comparison of performance parameters in the form of figures and tables, designs are also compared through line diagrams. Post-processing has been done on the final optimized population by studying the variation of different parameters with respect to objective functions. A criterion for the choice of one of the best design based on the minimum weight of bearing showing optimum copper loss.
\end{abstract}

\section{Keywords}

Radial Magnetic Bearings, Genetic Algorithms, Optimum Design, Multi-Objective Optimization

\section{INTRODUCTION}

In magnetic bearings, the transformation of energy takes place in two steps. First, the electrical energy transformed into the magnetic energy, and then it transforms into the mechanical energy. An AMB provides a non-contact means of supporting a rotating shaft through an attractive magnetic levitation force and hence they offer many advantages over conventional bearings. Active magnetic bearings are typical mechatronic product. They are composed of mechanical components combined with electronic elements such as sensors, power amplifiers and controllers which may be in the form of a microprocessor. As shown in figure1.besides the controller, the general control system also includes the sensor, $\mathrm{A} / \mathrm{D}$ and $\mathrm{D} / \mathrm{A}$ conversion and power amplifier. The rotor displacement along one of the axes is detected by these position sensors and converted into signals of standard voltage. Then compared with the setting value, the error signal enters the controller. After A/D conversion, the controller processes this digital signal according to a given regulating rule (control arithmetic) and generates a signal of current setting. After D/A conversion, this current signal enters the power amplifier, whose function is to maintain the current value in the electric magnet winding at the current level set by the controller. Therefore, if the rotor leaves its center position, the control system will change the electromagnet current in order to change its attraction force and, respectively, draws the rotor back to its balance position.

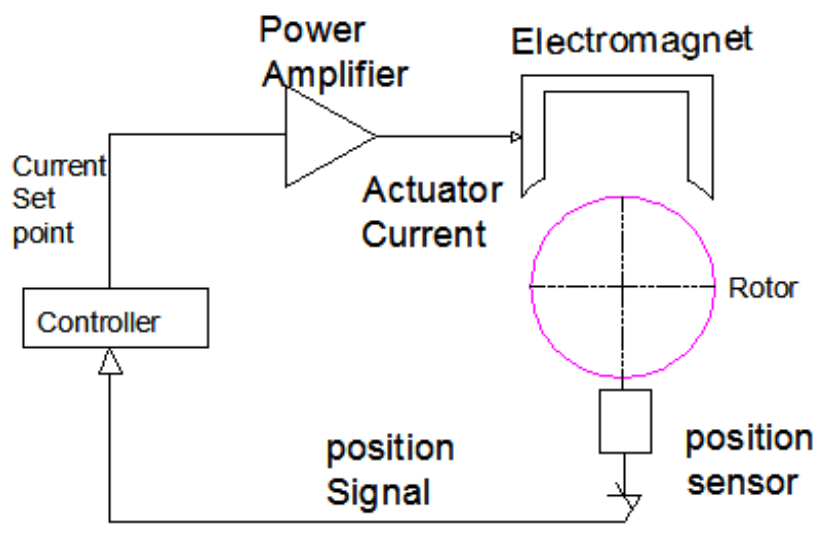

Fig 1: Block diagram of AMB system

In the present paper, a multi-objective optimization procedure of magnetic bearings have been presented and illustrated. Two objective functions have been considered, namely the minimization of copper loss and the minimization of weight to decide optimum number of poles for constant electromagnetic force to lift the rotor.

Bakay L., and Dubois M., [1] studied effect of $\mathrm{Cu}$ and Iron losses of optimized eight pole radial AMB on discharge time of no load long term flywheel energy storage. NSSN configuration is used. It concludes that for high discharge time for low loss AMB mass is smaller than in case of low discharge time. Optimal solution is for class of sinusoidal force signal. Also presented static allocation strategy for suboptimal power loss. Bakay L., Dubois M., and Ruel J.,[2] optimized AMB to minimize $\mathrm{Cu}$ and Iron loss for different magnitude of external force. Subsequently, Bloodgood et al. [3] applied the theory for the deterministic optimal design of a thrust magnetic bearing with bias permanent magnets. The magnetic bearing design included the determination of geometries of the bearing and the current supplied to carry a specified load for a specified gap.

Optimal design was carried out in two steps, including modeling the magnetic circuit, which determines the accuracy of achieving the requirement, and optimization of the design, which determines the efficiency of achieving the requirement [3]. Optimal design by using Gas of radial active magnetic bearings integrated with their control was studied by Chang and Chung [4]. Many techniques of multiobjective genetic 
algorithms (MOGAs) have been developed in recent years [7, 9, 11] among which Nondominated Sorting Genetic Algorithm-II (NSGA-II) [8] is one of the widely used technique $[5,10,12]$. Rao and Tiwari $[6,13]$ attempted to optimize the physical parameters of design of thrust magnetic bearings and rolling element bearing by using single objective genetic algorithms. The design optimization was performed by taking two objective functions, namely minimization of the power loss and maximization of the load to weight ratio, independently. The designs were compared for the cases with and without permanent magnets. Various approaches have been developed to aid the design of magnetic bearings. Parametric and systematic approaches have been developed to optimize the power to weight ratio of magnetic bearings [14] and to achieve minimum power. These approaches are useful in developing design strategies. Ying GAO, Lei Shi, Pinging Yao[15]presented new form of MOGA,steady state non dominated sorting genetic algorithm(SNSGA) is realized by combining steady state idea in single objective genetic algorithm(SOGA) and fitness assignment strategy of nondominated sorting genetic algorithm. R.T.marler, J.S.Arora [16] suggested that decision making can be done on the basis of 'a priori' or posteriori approach. In this method one needs prior knowledge of trade-off for taking decision. The different designs are generated as a Pareto-frontier and the designer can take a decision of choice depending on the different trade-offs generated. Tomoharu Nakashima, Hisao Ishibuchi [17] examines ability of genetic algorithm to find compact reference set nearest neighbor classification. In this case two objective are to maximize classification of reference set and to minimize size of reference set. Deepti Chafekar, Liang shi, Jiang Xuan [18] proposed novel method for solving multiobjective optimization problem using reduced models. Santosh shelke, R.V.Chalam [19, 21] proposed theoretical design of eight pole radial magnetic bearing and copper loss optimization.E.H.maslen [20] discussed fundamentals and optimum design of radial and thrust magnetic bearing.

\section{GEOMETRY of RADIAL MAGNETIC BEARING}

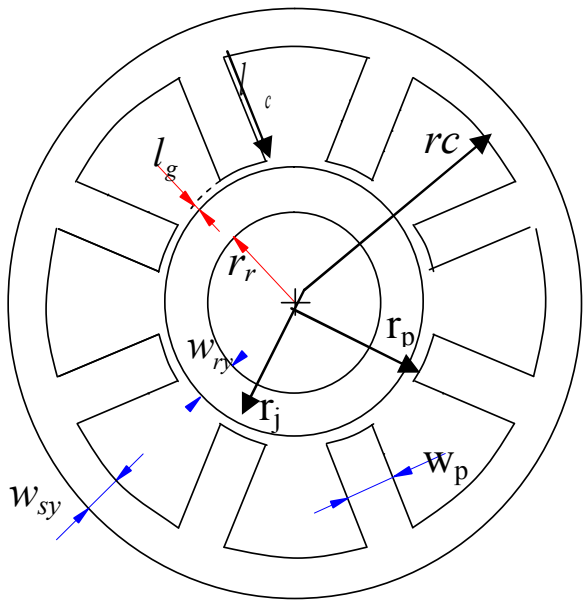

Fig 2: Radial Magnetic Bearing

Where,

$l_{g}$ - Air gap, $t_{c}$-Thickness of coil, $l_{c}$-radial length of coil on pole, $r_{c}$-coil space radius, $r_{j}$-Radius of journal, $r_{r}$-Radius of rotor, $r_{p}-$ Pole tip radius, $w_{s y}-$ Width of stator

yoke, $w_{r y}-$ Width of rotor yoke. Gap area, $A_{g}=w_{p} \cdot l_{p}$, $w_{p}$-width of pole, $l_{p}$-length of pole, $n_{p}$-Number of poles, For available coil space thickness of coil is calculated as,

$$
t_{c}=r_{p} \tan \left(\frac{\pi}{n_{p}}\right)+\left(\frac{w_{p}}{2}\right)
$$

We know that maximum force, $F_{\max }$ carried by AMB

$$
F_{\max }=\left(B_{\text {sat }}^{2} n_{p} A_{g} / \mu_{0}\right)
$$

\section{MULTI-OBJECTIVE OPTIMIZATION}

The multi-objective optimization problem consists of objective functions to be optimized by design variables while satisfying certain constraints.

\subsection{Bounds for Variables}

Table 1. Choice of design vectors.

$$
\begin{aligned}
& r_{c_{\text {min }}}=0.3 m, r_{c_{\text {max }}}=0.8 \mathrm{~m} ; \\
& r_{p_{\text {min }}}=0.03 m, r_{p_{\max }}=0.08 \mathrm{~m} ; \\
& l_{c_{\text {min }}}=0.2 m, l_{c_{\max }}=0.8 \mathrm{~m} ; \\
& n_{p_{\text {min }}}=4, n_{p_{\max }}=12 ;
\end{aligned}
$$

\subsection{Input Parameters}

Table 2. Constant parameters [18] and inputs assumed for radial magnetic bearing design

Permeability of vacuum, $\mu_{0}=4 \pi \times 10^{-7} \mathrm{H} / \mathrm{m}$;

Resistivity, $\rho=2 \times 10^{-5} \Omega \mathrm{m}$;

Air gap length, $l_{g}=0.001 \mathrm{~m}$;

Radius of journal, $r_{j}=0.03 \mathrm{~m}$;

Width of stator, $w_{s y}=0.01 \mathrm{~m}$;

Saturated flux density, $B_{\text {sat }}=1.2 T$;

flux density, $B_{\mathrm{min}}=0.2 \mathrm{~T}$;

Coil mmf loss factor, $K_{i}=1.394$;

Actuator loss factor, $K_{a}=1.072$;

Coil packing factor, $\eta=0.85$;

Electromagnetic force, $F=350 N$;

Maximum volume of coil, $V_{\max }=0.00039 \mathrm{~m}^{3}$;

Maximum copper loss, $P_{\max }=500 \mathrm{~W}$;

Iron saturation factor, $\alpha=0.5$;

Weight densities, $\rho_{s}=7770 \mathrm{~kg} / \mathrm{m}^{3} ; \rho_{c}=8910 \mathrm{~kg} / \mathrm{m}^{3}$;

Current density, $J_{u b}=600000 \mathrm{~A} / \mathrm{m}^{2}$;

\subsection{Objective Function}


When the genetic algorithm is applied to two objective functions, namely the copper power-loss in the magnetic coil and the weight of the magnetic bearing, it is observed that these are mutually conflictive [6], i.e. with the decrease in the power-loss causes an equivalent increase in the weight and visa versa.

\subsubsection{Power loss in coil}

From fundamental relation in magnetic, bearing flux density in terms of coil current is given by,

$$
B=\frac{\mu_{0} n i}{2 l_{g}}
$$

Where,

$n i$ is the magneto- motence and $\mu_{0}$ is the permeability of the vacuum.

Groom and Bloodgood [3] proposed a model by adding coil $\mathrm{mmf}$ loss factor, $K_{i}$ and actuator loss factor, $K_{a}$ to ideal model.

$$
B=\frac{\mu_{0} K_{i} n i}{2 K_{a} l_{g}}
$$

The magnetic force exerted by the actuator could be expressed as

$$
F=\frac{B A_{g}}{\mu_{0}}
$$

The standard form of expression of the power-loss in the coil is given as

$$
\begin{gathered}
P=i^{2} R \\
P=\rho \eta J^{2} A_{c} V_{c}
\end{gathered}
$$

The cross sectional area and the volume of the coil are expressed as

$$
A_{c}=t_{c}\left(r_{c}-r_{p}\right) \text { and } V_{c}=A_{c} l_{c}
$$

\subsubsection{Weight of magnetic bearing}

The overall weight of the magnetic bearing could be expressed as

$$
W_{\text {Total }}=W_{c}+W_{s}
$$

Where,

Weight of coil, and stator is

$$
\begin{aligned}
& W_{c}=\rho_{c} V_{c} \text { And } W_{s}=\rho_{s} V_{s} \\
& V_{s}=\pi\left(r_{s}-r_{c}\right) w_{s y}+\left(r_{c}-r_{p}\right) A_{g}
\end{aligned}
$$

\subsection{Constrains}

The maximum and minimum forces exerted by the bearing, $F_{\max }$ and $F_{\min }$, are determined from the current stiffness and the displacement stiffness at corresponding gaps, and these form equality constraints [3]. Recall equation (4) and (5) to express electromagnetic force are

$$
F_{\text {max }, \text { min }}=\left(0.25 \mu_{0} A_{g}\right)\left(\frac{K_{i} n_{p} i_{\text {max }, \text { min }}}{K_{a} l_{g_{\text {max }, \text { min }}}}\right)^{2}
$$

$$
l_{g_{\text {max }, \text { min }}}=\left(l_{g} \pm x_{\max , \min }\right)
$$

Maximum displacement of rotor in terms of force and displacement stiffness,

$$
x_{\max }=\left(F_{\max }-F\right) / K_{x}
$$

The current density supplied in the coil should not be more than the maximum current density that the coil material can sustain, which is called the coil saturation current density.

The maximum and minimum magneto-muteness required could be found and then current densities required to be supplied in the coil is

$$
n_{p} i_{\max , \min }=\eta A_{c} J_{\max , \min }
$$

From equation (12) and (15) we get,

$$
J_{\text {max }, \text { min }}=\left(\frac{K_{a} l_{g_{\text {max } \text { min }}}}{K_{i} \eta A_{c}}\right) \sqrt{\frac{4 F_{\text {max }, \text { min }}}{\mu_{0} A_{g}}}
$$

Hence, constraint becomes,

$$
J_{\text {sat }} \geq J_{\text {max }} ; J_{\text {min }} \geq 0
$$

The magnetic flux density in the stator-iron should not be more than the iron magnetic saturation flux density. The maximum and minimum magnetic flux densities of the stator that are required to support the maximum and minimum loads are expressed as,

$$
B_{\max , \min }=\sqrt{\mu_{0} F_{\max , \min } / A_{g}}
$$

From above it can be observed that for a fixed maximum force, as the pole-face area of the air-gap increases, the maximum magnetic flux density required in the stator-iron reduces.

For a linear range of operation the actuator could be designed to have the magnetic flux density between two limits. In this work an iron saturation factor, $\alpha$, is used to express the linear range as a percentage of the magnetic saturation flux.

According to Equation (4), the linear range limits [4] for lower and upper bounds are expressed as

$$
\frac{\mu_{0} K_{i} n i_{\mathrm{min}}}{2 K_{a} l_{g_{\mathrm{min}}}} \geq \alpha_{\mathrm{min}} B_{\text {sat }}
$$

and

$$
\frac{\mu_{0} K_{i} n i_{\max }}{2 K_{a} l_{g_{\max }}} \leq \alpha_{\max } B_{\text {sat }}
$$

Equations (19) and (20) constrain the choice of $n i_{\max }$.

The choice of $n i_{\max }$ influences the cross sectional area of the coil. As the thickness of coil increases, the available thickness of the iron core decreases which in turn increases the magnetic flux density for supporting a fixed load. Thus, equations (19) and (20) influence the choice of design parameters, i.e. the inner and outer radii of the coil.

Where, 
Table 3. The summary of the formulation of the magnetic bearing design for the multi-objective optimization

Minimize $f_{i}(x)=1,2$

Where,

$\mathrm{x}=\left\{r_{c}, r_{p}, l_{c}, n_{p}\right\}$

Subject to;

$g_{i}(x) \geq 0 ; j=1,2 \ldots \ldots, 6$;

$h_{k}(x)=0, k=1,2 ; x^{L} \leq x_{p} \leq x^{U} ; p=1, \ldots, 4$.

Where,

$f_{1}(x)=p_{c u} ; f_{2}(x)=W_{\text {Total }}$.

$g_{1}(x)=J_{\text {sat }}-J_{\max }$;

$g_{2}(x)=J_{\text {min }}$;

$g_{3}(x)=\alpha_{\max } B_{\text {sat }}-B_{\max } ;$

$g_{4}(x)=B_{\text {min }}-\alpha_{\text {min }} B_{\text {sat }} ;$

$g_{5}(x)=V_{\max }-V_{c}$;

$g_{6}(x)=P_{\max }-P$;

$h_{1}(x)=F_{\max }-F\left(l_{g_{\max }}, i_{\max }\right)$;

$h_{2}(x)=F_{\min }-F\left(l_{g_{\min }}, i_{\min }\right)$;

\section{RESULTS AND DISCUSSIONS}

The non-dominated sorting genetic algorithm-II (NSGA-II) [8] is implemented to the present work. The algorithm involves the single point crossover and the bitwise mutation for binary variables, the simulated binary crossover (SBX) and the polynomial mutation for real coded variables, and the parameter-less approach is used to handle constraints.

For the optimization, SBX parameters namely the probability of the crossover, the probability of the mutation, the crossover distribution index, the mutation distribution index and basic seed are assumed to be $0.1,0.01,5$, and $10,0.1$ respectively, where the number of real variables are 4 for the present problem. To have better understanding of the behavior of design variables with respective to objective functions in the final optimized population, a population of 200 is taken with 1000 generations. Objective functions are optimized at the operating point of magnetic bearings. The Pareto front [6] is the locus of different nondominated solutions in the multi-objective space. The nondominated sorted genetic algorithm gives a group of solutions, which are ranked according to the degree to which constraints are satisfied and the objectives are optimized. Final solutions optimize objective functions to different degrees. For a weighted sum approach of the single objective optimization, it gives a single optimum value for each run that may not be Pareto optimal. But in the case of multi-objective optimization, we get different Pareto optimum designs in a single run. From this group of solutions the designer chooses proper design according to the application that is intended.

There are two types of constraint handling, i.e., the hard and the soft. In the case of hard, the violation of the constraint is not tolerated; in the case of soft constraint the violation of the constraint is tolerated to a specified degree In the present case, the equality constraint of load to be supported in Equation (12) is satisfied by determining the required current densities and flux densities from Equations(16), 18) . All the other constraints in Equations (17), (19), (20), are greater than or equal to type, and all the constraints are treated to be hard.

Table 4. Initial Bounds of the design variables

\begin{tabular}{lccccc}
\hline \multicolumn{5}{c}{ Initial bounds } \\
\hline Variables : & $r_{c}(\mathrm{~m})$ & $r_{p}(\mathrm{~m})$ & $l_{c}(\mathrm{~m})$ & $n_{p}$ \\
\hline Lower limit: & 0.3 & 0.03 & 0.2 & 4 \\
Upper limit: & 0.8 & 0.08 & 0.8 & 12 \\
\hline
\end{tabular}

Table 5. Final Bounds of the design variables

\begin{tabular}{lcccc}
\hline & \multicolumn{4}{c}{ Final bounds } \\
\hline Variables : & $r_{c}$ & $r_{p}$ & $l_{c}$ & $n_{p}$ \\
\hline Lower limit: & 0.306 & 0.0305 & 0.2 & 7 \\
Upper limit: & 0.78 & 0.0416 & 0.208 & 10 \\
\hline
\end{tabular}

Table 6. Optimum bearing variables after final population.

\begin{tabular}{lcccc}
\hline Variables : & $r_{c}$ & $r_{p}$ & $l_{c}$ & $n_{p}$ \\
\hline Optimum: & $0.7109 \mathrm{~m}$ & $0.0351 \mathrm{~m}$ & 0.204 & 8 \\
\hline
\end{tabular}

\section{Table 7. Optimum dimensions of RMB}

$$
\begin{aligned}
& A_{c}=0.00167 \mathrm{~m}^{2}, \\
& A_{g}=0.003398 \mathrm{~m}^{2}, \\
& V_{s}=0.000833 \mathrm{~m}^{3} \\
& V_{c}=0.000334 \mathrm{~m}^{3}, \\
& W_{s}=6.474 \mathrm{Kg}, \\
& W_{c}=2.9615 \mathrm{Kg}
\end{aligned}
$$

The minimum and maximum values of the theoretical feasible bounds on the design vector and final optimized solution are given in Table 4 to 7 respectively. It could be noted that the bounds obtained by GA are within the range of theoretical bounds. From Table 5,the optimum ranges (in the final population after 1000 generations) on the coil space radius, pole tip radius, coil length; number of poles are 94.8\%, 22.2\%, 13.3\% and $12.5 \%$ of feasible ranges, respectively. The optimum ranges of the pole tip radius, coil length are near the lower limit of the feasible range and except the coil space radius. 


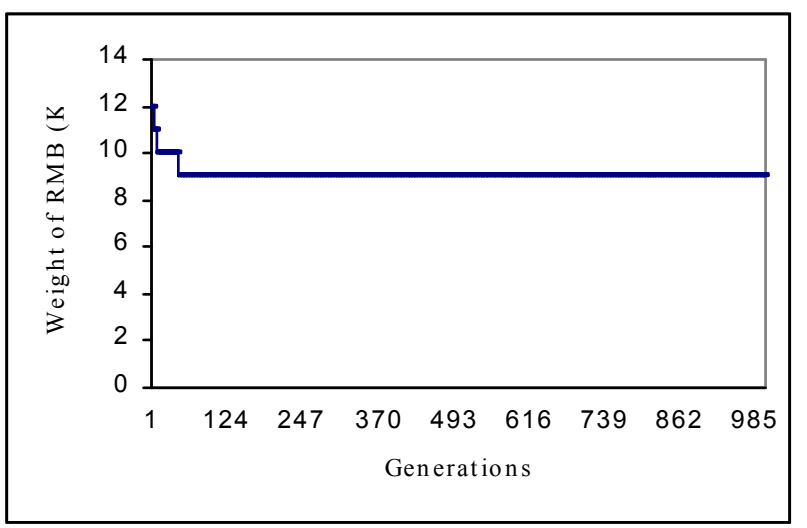

Fig 3: Convergence graph for minimum weight of RMB

Convergence of the minimum weight with the generation is plotted in Figure 3.

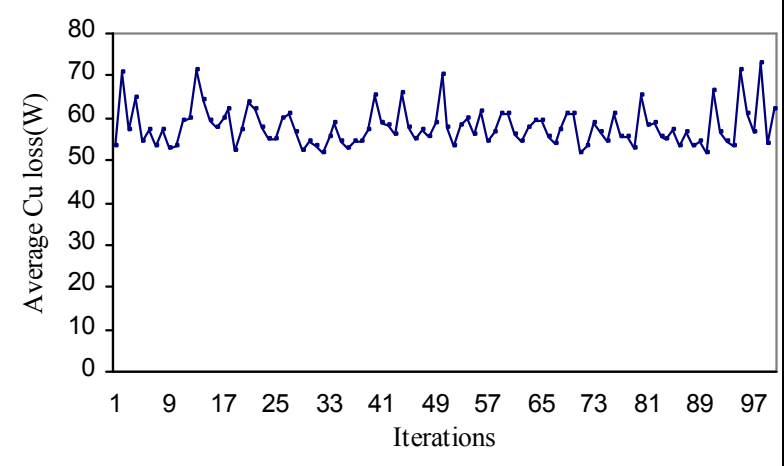

Fig 4: Average power loss for 100 runs

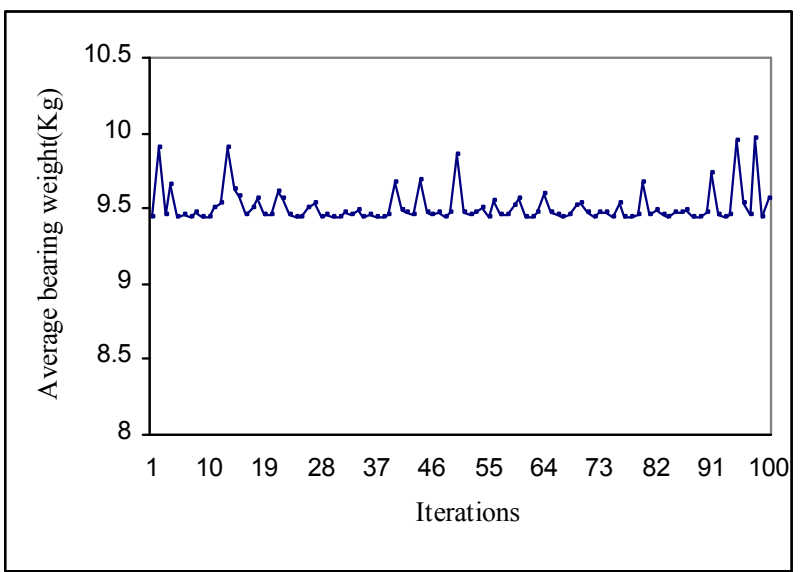

Fig 5: Average bearing weight for 100 runs

Results have been obtained with population size of 200 and 100 runs for 1000 generations. From figure 6 it is observed that the decrease in the power-loss causes an equivalent increase in the weight and visa versa.

Figure 6. Shows that optimum weight of bearing is $9.435 \mathrm{Kg}$ where as optimum copper loss is $54.635 \mathrm{~W}$.

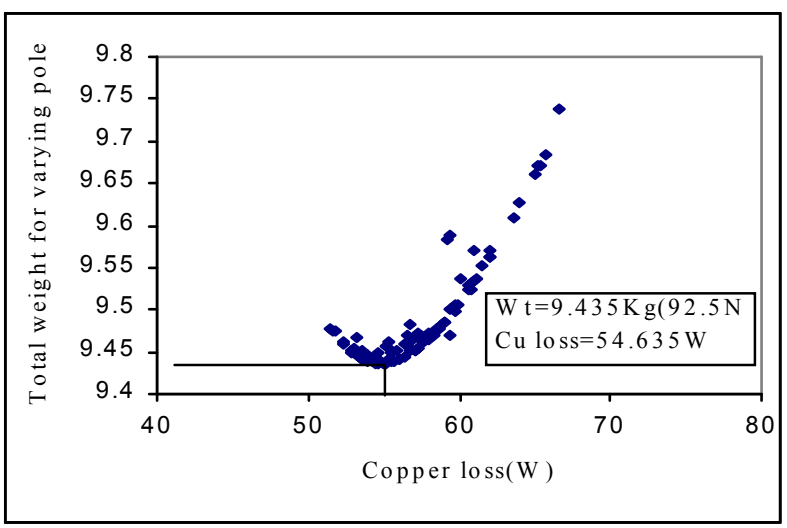

Fig 6: copper loss versus bearing weight

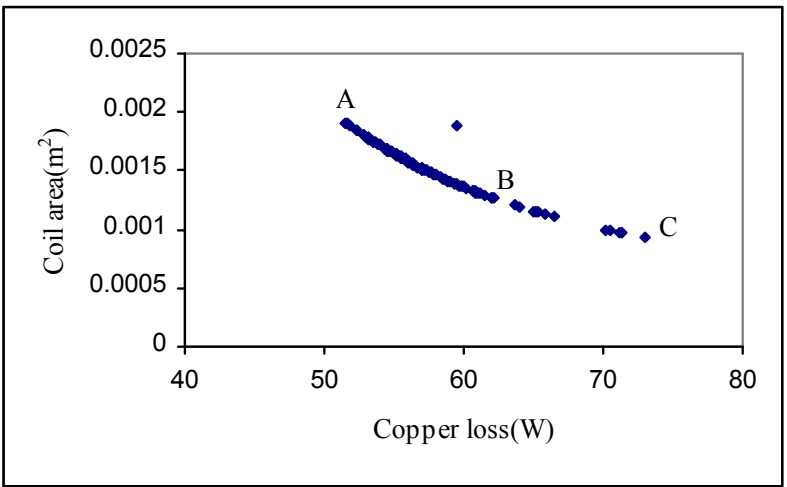

Fig 7: Effect of coil area on copper loss

As shown in Figures 7, the cross sectional area of the coil decrease with the power-loss to a minimum limit, until the saturation limit of the current density is reached. Between points $\mathrm{B}$ and $\mathrm{C}$ of curve, solution points are scattered than between points A and B.

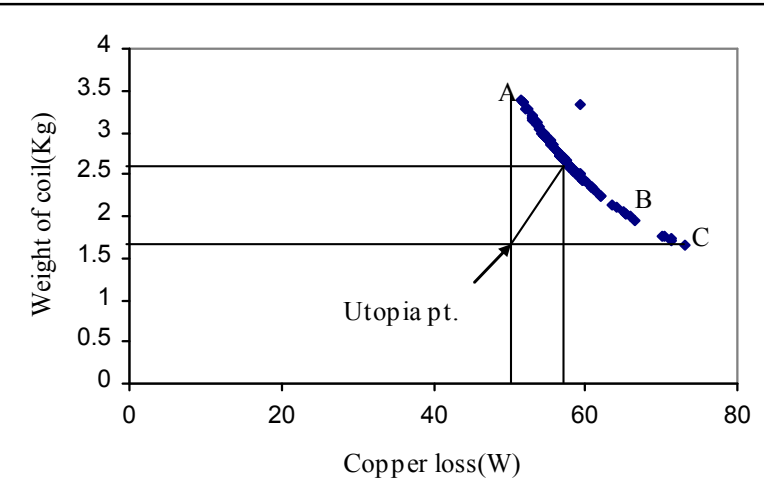

Fig 8: (a) Effect of scattered region on bearing geometry.

Figure 8(a) shows procedure to select optimized bearing geometry from the paretofront.Here we obtained copper loss $55 \mathrm{~W}$ and weight of coil is $2.6 \mathrm{Kg}$.where as optimum values obtained at run 93 for copper loss $54.63 \mathrm{~W}$, weight of coil $2.96 \mathrm{Kg}$, weight of stator $6.47 \mathrm{Kg}$. Hence neglecting scattered region $\mathrm{B}-\mathrm{C}$ and applying pareto-front for region A-B, we get accuracy for optimum values of bearing geometry as shown in figure $8(\mathrm{~b})$. When observed A-C, optimum design of the minimum normalized distant member, an increase of $23 \%$ in the power- 
loss generated a decrease of $72.2 \%$ in the weight, where the percentage of change is determined according to the formula for Percent of change of quantity=

$=\left(\frac{X-X_{\min }}{X_{\max }-X_{\min }}\right) \times 100$

Where,

$\mathrm{X}$ represents the quantity (i.e., the copper loss or weight) considered.

$X_{\max }$ and $X_{\min }$ are the maximum and minimum values of the corresponding quantities in the cases of minimum weight and minimum power-loss.

The weight of the coil versus copper loss shows similar trends as the area of cross section before point $B$, but after point $B$ the decrease in weight with the Equation (7).

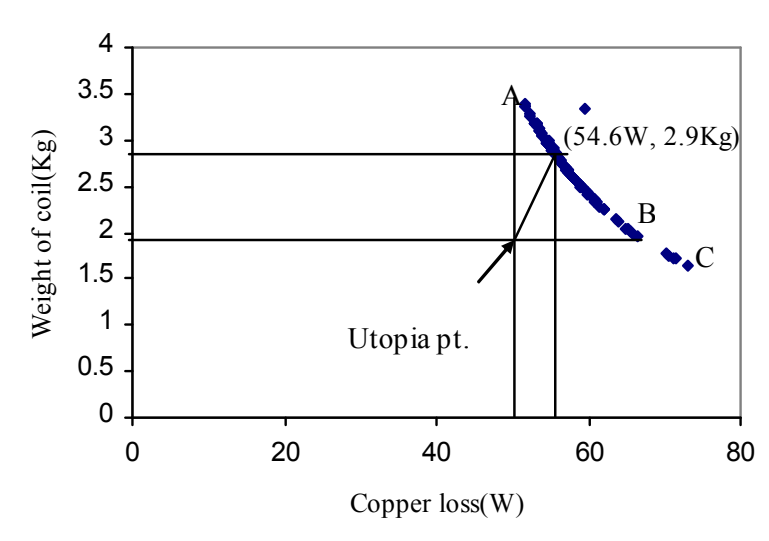

Fig 8 (b): Optimum bearing geometry using pareto-front.

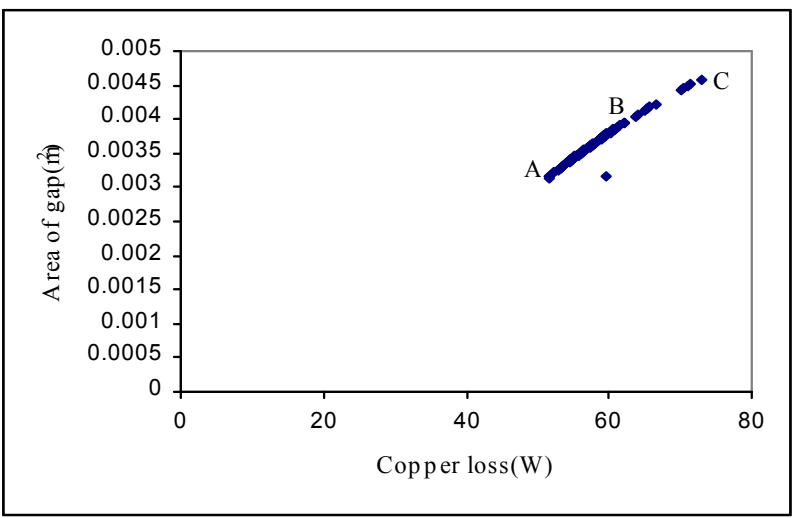

Fig 9: Effect of area of gap on Copper loss

As shown in Figures 9, the copper loss increases with increase in area of gap, until the saturation limit at point $\mathrm{C}$ of the current density is reached. Between points $\mathrm{B}$ and $\mathrm{C}$ of curve, solution points are scattered than between points $\mathrm{A}$ and $\mathrm{B}$. Figure 10 shows increase in coil area volume of coil increases which is in directly proportional to weight of coil.

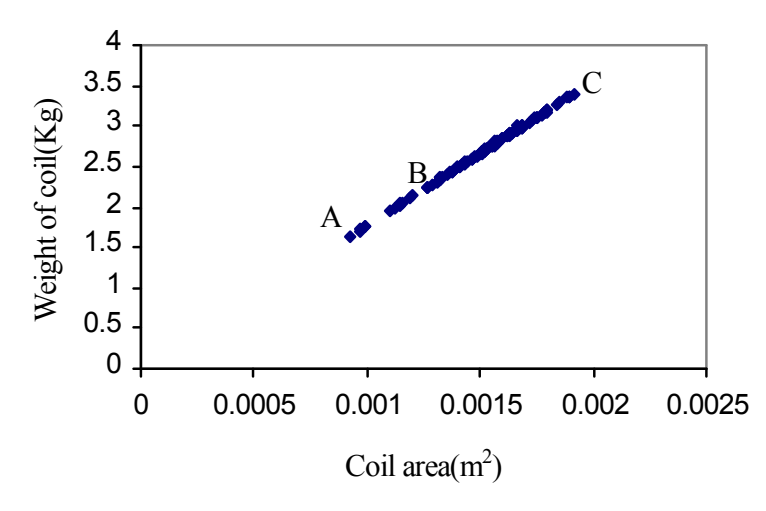

Fig 10: Effect of weight of coil on copper loss

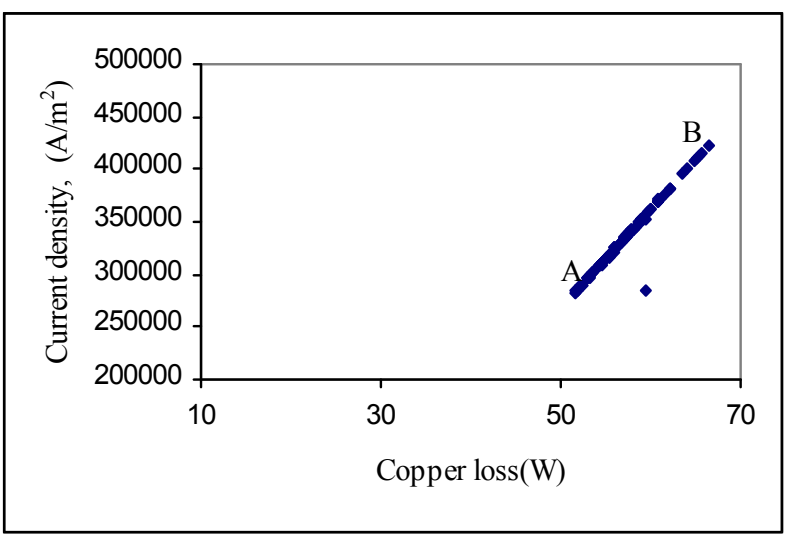

Fig 11: Effect of current density on copper loss

Figure 11 shows that copper loss is accompanied by the increase in the current density, which linearly increases up to its saturation limit (i.e., point B) power-loss.

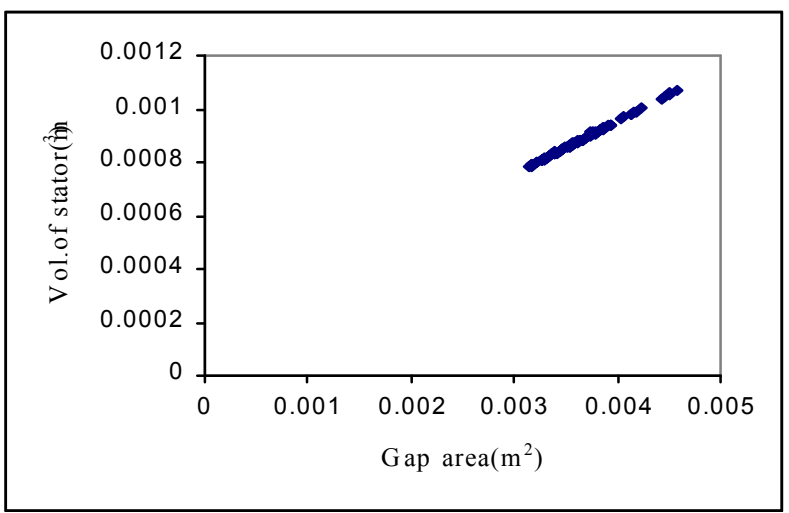

Fig 12: Effect of gap area on volume of stator

Figure 12 shows change in dimensions of pole affect pole face area i.e. gap area. This means increase in pole size along axis of rotor, increases pole face area or gap area due to which volume of stator increases proportionally. 


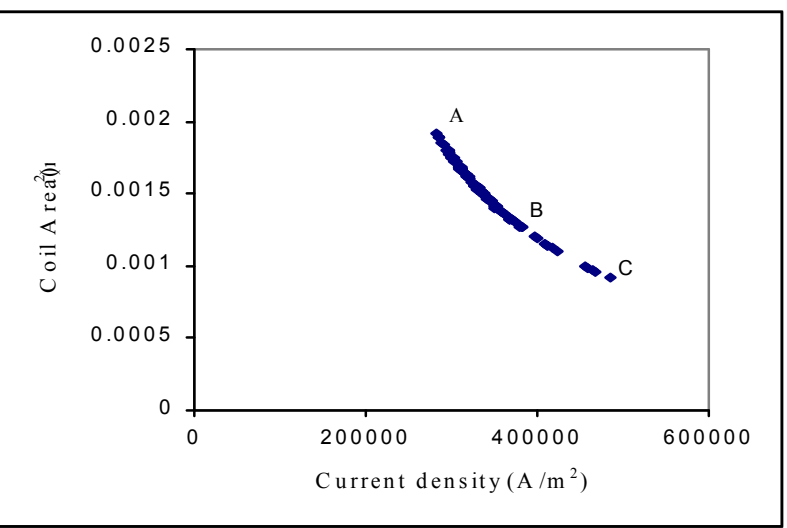

Fig 13: Effect of coil area on current density

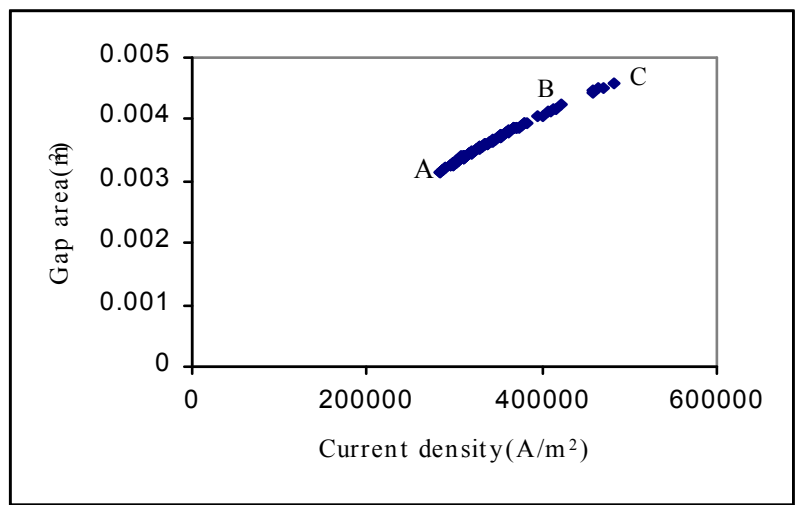

Fig 14: Effect of gap area on current density

The constraint violation plots in the final population (i.e., after 1000 generations) are shown in Figure 13and 14.Constraints used are greater than or equal to type, and as all the values are above zero for all the solutions are feasible solutions. Curve A to B is for unscattered solution and limiting values located at $\mathrm{A}$ and $\mathrm{B}$ are feasible for finding optimum values of gap area and coil area using pareto-front method.

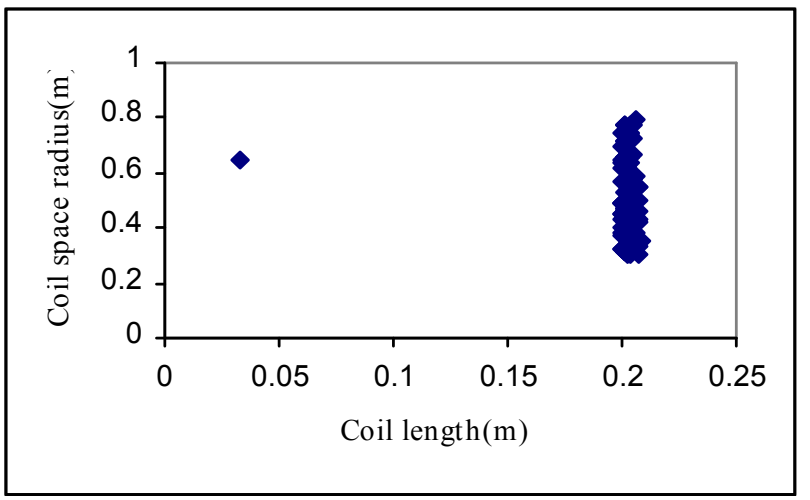

Fig 15: Coil length versus coil space radius

Figure 15 shows for final population after 100 runs, optimum bounds for coil length and coil space radius are $0.2-0.21 \mathrm{~m}$ and 0.3 $0.78 \mathrm{~m}$.

Figure 16 shows all solution points are within pole limit 7 to 10 . Some scattered solution points in upper region but lower region is dense with convex curve shape From final bound of poles 8 number of poles are suitable for avoiding cross coupling effect in radial magnetic bearing. With this curve we get optimum bearing weight $9.43 \mathrm{Kg}$ for optimum number of 8 poles

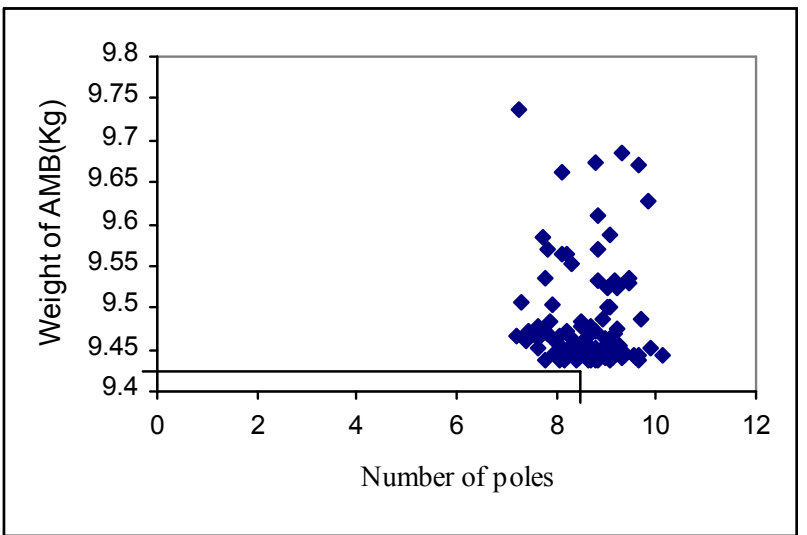

Fig 16: Effect of varying pole on weight of bearing

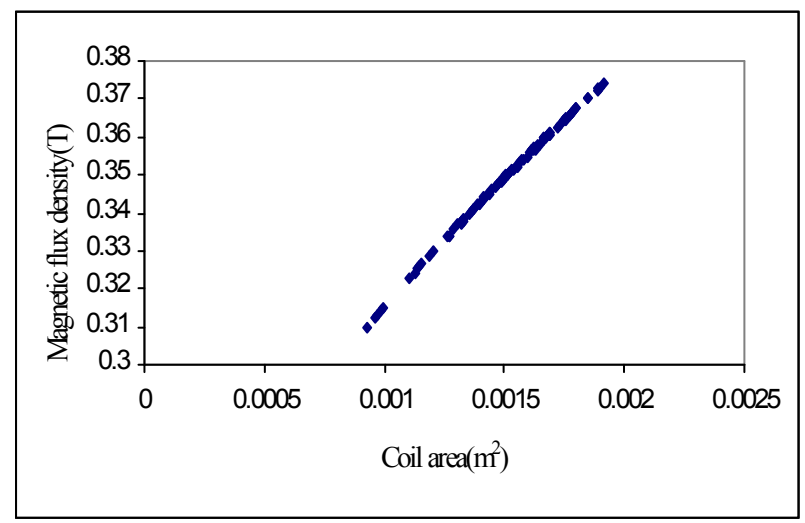

Fig 17: Effect of Magnetic flux density on coil area

Figure 17 shows magnetic flux density increases with increase in coil area.

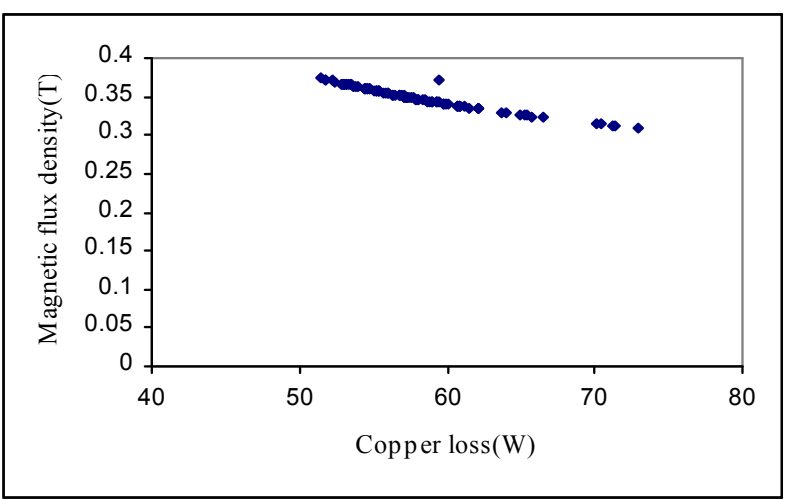

Fig 18: Copper loss versus Magnetic flux density

Once the magnetic flux density is saturated as shown in Figure 18 , the magnetic flux density is slightly decreases with increase in the power-loss.

\section{CONCLUSION}

In this present work, the optimal design of radial active magnetic bearings using MOGAs has been carried out. Objective functions and associated constraints have been presented and discussed. Two objectives have been considered, namely the minimizing the copper loss and weight of bearing. Coil dimensions and 
number of poles have been proposed as design variables based on their dependency on objective functions. The geometries of the designs with optimal individual objectives and a chosen design from the optimal Pareto front based on the minimum normalized distance criterion are compared. Performance analysis is carried out graphically for bearing geometry and variables. The behavior of final optimized population designs has also been studied. Copper loss, weight of bearing, magnetic flux density and coil current density are observed for different design parameters. Moreover, in the final population after 1000 generations, the optimum range of variables on the coil space radius, pole tip radius, coil length, number of poles are $94.8 \%, 22.2 \%, 13.3 \%$ and $12.5 \%$ of feasible ranges, respectively. The optimum ranges of the pole tip radius, coil length are near the lower limit of the feasible range and that of the coil space radius is near the upper limit. In optimum design of the minimum normalized distant member, an increase of $23 \%$ in the power-loss generated a decrease of 72 . $2 \%$ in the coil weight.

From graphs it is observed that objective functions are mutually conflictive i.e. with the decrease in the power-loss causes an equivalent increase in the weight and visa versa.Otimum value of objective functions are $54.63 \mathrm{~W}$ and $9.43 \mathrm{Kg}$.

\section{ACKNOWLEDGEMENT}

Corresponding author would like to thank Prof.R.V.chalam for his valuable guidance for this research in organization National Institute of technology, Warangal, India.

\section{REFERENCES}

[1] Bakay L., and Dubois M., "Losses in an optimized 8-pole radial magnetic bearing for long term flywheel energy storage", IEEE transaction on magnetic, Canada, 2007,3-5.

[2] Bakay L., Dubois M., and Ruel J, "Mass-Loss relationship optimized 8-pole AMB for long term flywheel energy storage", IEEE AFRICON, Kenya, 2009,.2-3.

[3] V.D. Bloodgood, Jr., N.J. Groom, C.P. Britcher, "Further Development of an Optimal Design Approach Applied to Axial Magnetic Bearings", NASA-2000-7ismb-vdb.

[4] H. Chang, S.C. Chung, "Integrated Design of Radial Active Magnetic Bearing Systems using Genetic Algorithms, Mechatronics", vol. 12(1), 2002, 19-36.

[5] S. Ghanmi, M. Guedri, M.-L. Bouazizi, N. Bouhaddi, Use of Metamodels in the Multi-Objective Optimization of Mechanical Structures with Uncertainties, International Journal for Computational Methods in Engineering Science and Mechanics, vol. 8(5), 2007, 283-302.

[6] Jagu S.Rao, Rajiv Tivari, "Optimum design and analysis of thrust magnetic beating using genetic Algorithm", International Journal for Computational Methods in Engineering Science and Mechanics, vol. 9, 2008, 223-245.
[7] C.A.C. Coello, "An Updated Survey of GA-Based Multiobjective Optimization Techniques," ACM Computing Surveys, vol.32 (2), pp.109-143, 2000.

[8] K. Deb, A. Pratap, S. Agarwal, T. Meyarivan, "A Fast and Elitist Multiobjective Genetic Algorithm”, NSGA-II, IEEE Trans. Evol. Comput, vol. 6, 2002, 182-197.

[9] C.M. Fonseca, P.J. Fleming, "An Overview of Evolutionary Algorithms in Multi-objective Optimization, Evolutionary Computation", Springer, vol. 3(1), 1995, 1-16.

[10] L.T. Bui, J. Branke, H.A. Abbas, "Multiobjective Optimization for Dynamic Environments," IEEE Paper No. 0-7803-9363-5/05, 2005, 2349-2356.

[11] Y. GAO, L. Shi, P. Yao, "Study on Multi-Objective Genetic Algorithm", Proc.3rd World Cong. On intelligent Control and Automation, 2000, 646-650.

[12] X. Hu, Z. Huang, Z. Wang, "Hybridization of the MultiObjective Evolutionary Algorithms and the Gradient-based Algorithms", IEEE Paper No.0-7803-7804-0 /03, 2003, 870-877.

[13] B.R.Rao, R.Tiwari, "Optimum design of rolling element bearing using genetic algorithm," Mechanism and machine theory, 2007, 233-250.

[14] C.L. Malone, "Power to Weight Optimization for Magnetic Bearings," Magnetic Bearings, Magnetic Drives and Dry Gas Seals Conference \& Exhibition, Alexandria, Virginia, July, 1993.

[15] Ying Gao, Lei Shi, Pinging Yao, "Study on Multiobjective Genetic Algorithm," IEEE transaction on Intelligent control and Automation, China, 2000, 646-650.

[16] R.T.marler, J.S.Arora, "Survey of multiobjective optimization methods for engineering," Springer,Vol 26,2004,369-395.

[17] Tomoharu Nakashima, Hisao Ishibuchi, "Genetic algorithm based approaches for finding the minimum reference set for nearest neighbor classification,IEEE, 1998,709-714.

[18] Deepti Chafekar, Liang shi, Jiang Xuan, "multiobjective genetic algorithm optimization using reduced models, IEEE transaction on system, Vol 35(2), 2005, 261-265.

[19] Santosh shelke, R.V.Chalam, "Optimum energy loss in electromagnetic bearing”, IEEE, vol.3, 2011,.374-378.

[20] E.H.Maslen, "Text book on Magnetic Bearings, University of Virginia, 36(4), 2000, 1009-1013.

[21] Santosh shelke, R.V.Chalam, "Optimum copper loss analysis of eight pole radial magnetic bearing: Multi objective genetic algorithm", accepted for Springer, Proceeding of International conf. ccpe 2011. 